\title{
变截面微纳米通道内聚乙烯分子注射过程的 分子动力学模拟
}

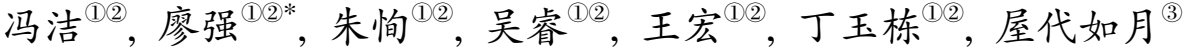 \\ (1) 重庆大学工程热物理研究所, 重庆 400030; \\ (2) 低品位能源利用及系统教育部重点实验室, 重庆 400030; \\ (3) 神户大学机械工学部, 神户 657-8501 \\ *联系人, E-mail: 1qzx @cqu.edu.cn
}

2010-09-26 收稿, 2010-11-17 接受

国家自然科学基金资助项目(50876119)

摘要 采用分子动力学方法模拟了变截面微纳米通道内聚乙烯分子的注射过程, 分析了微纳米 通道截面结构及外加作用力对注射过程中聚乙烯分子流变和结构特性的影响. 研究结果表明: 注射流动过程中, 通道壁面附近存在粒子吸附层, 且吸附层厚度随通道锥面倾角增大而增大; 聚乙烯分子链的注射距离随锥面倾角增大而减小、随外加作用力增大而增大; 在锥面倾角为 $\alpha=45^{\circ}$ 的通道中聚乙烯分子均匀填充整个通道, 且沿流动方向上出现单轴拉伸现象, 在较大作

关键词

分子动力学

聚乙烯

注射

纳米通道 用力情况下, 该拉伸更为显著, 使得注射过程更容易完成.

随着纳米加工技术的发展，精确的分配和传输 技术在医药、机械、化工、生物工程等领域得到不断 发展, 如电磁微洜驱动的纳米注射器 ${ }^{[1]}$, 纳米注射的 毛细管气相色谱装置 ${ }^{[2]}$, 以及纳米通道中陶瓷体注射 的脱脂行为 ${ }^{[3]}$ 等微纳米注射技术引起了研究者的广 泛关注 ${ }^{[4,5]}$. 此外, 有机聚合物的喷射模塑法在很多 领域也得到重视和应用 ${ }^{[6,7]}$, 但因为拓扑学的限制, 聚合物流体在微纳米尺度流动中表现出独特的流变 性质 ${ }^{[8,9]}$, 且聚合物的注塑过程对溶液温度、黏度、注 射速度和注人压力等都有精确的要求, 任一参数的 变化均将导致聚合链流体性质的改变, 从而影响整 个注塑过程. 因此, 聚合物在微纳米尺度下的流动是 一个非常复杂的流动问题. 鉴于实验的难度, 分子动 力学模拟以其特有的优势在研究聚合物流体流变性 质方面成为了有效辅助研究工具, 并已经得到了广 泛的应用. Hess 等 ${ }^{[10 ~ 12]}$ 分别运用非平衡分子动力学 方法模拟了库特流动条件下聚合物的流体性质; 而 对复杂未知的聚合链几何结构, 文献中常用哑铃模
型 ${ }^{[13]}$ 、自由连接链模型 ${ }^{[14]}$ 和 Rouse 模型 $^{[15]}$ 来简化聚 合链，但这些简化模型在模拟过程中都有一定的局 限, 如哑铃模型极大地忽略了聚合物大分子内部众 多的自由度, 自由连接链模型和 Rouse 模型的简化很 多时候与实验观测不一致等, 因此掌握聚合物的特 性参数将有助于提高分子动力学模拟的准确性. 近 年来, Yashiro 等人 ${ }^{[16]}$ 获得了聚乙烯分子的特征数值, 而聚乙烯分子作为一种典型的高分子聚合物在众多 领域都得以应用，因此，本文将采用分子动力学方法 及原子基团模型 ${ }^{[17]}$ 对聚乙烯分子链在变截面纳米通 道中进行注射过程时的结构和流变性质进行模拟， 研究槽道结构及外力大小对聚乙烯流体注射过程的 影响规律.

\section{1 模拟方法及系统}

聚乙烯是通过乙烯 $\left(\mathrm{CH}_{2}=\mathrm{CH}_{2}\right)$ 加成聚合而成, 即由重复的亚甲基 $\left(-\mathrm{CH}_{2}-\right)$ 单元连接而成. 采用原子 基团模型 ${ }^{[17]}$ 模拟聚乙烯分子时，将每个亚甲基视为 
一个粒子, 从而可将聚乙烯分子链看成由多个粒子 组成, 如图 1 所示, 其中两个粒子间的距离为键长 $r$, 3 个粒子构成两条相交直线的角度为键角 $\theta, 4$ 个粒子 构成两个平面的夹角为二面角 $\phi$.

在采用分子动力学模拟过程中, 聚乙烯分子间 的势能为其键长能、键角能、二面角能和范德瓦尔势 能的总和, 用 $E_{\mathrm{tot}}$ 表示如下:

$$
E_{\text {tot }}=E_{b s}(r)+E_{b e}(\theta)+E_{t o}(\phi)+E_{v w}(\bar{r}),
$$

式中 $E_{b s}(r)$ 为键长 $r$ 的二体势能; $E_{b e}(\theta)$ 为键角 $\theta$ 的 三体势能; $E_{t o}(\phi)$ 表示二面角 $\phi$ 的四体势能; $E_{v w}(\bar{r})$ 表示距离为 $\bar{r}(\bar{r}<8 \mathrm{~nm})$ 的不相连粒子或同一条链上 相隔超过 4 个粒子的粒子之间范德瓦尔势能, 用 12-6LJ 型势能模型表示. 上述各势能函数分别计算 如下 ${ }^{[16]}$ :

$$
\begin{gathered}
E_{b s}(r)=\sum_{\text {node }}\left\{k_{r}\left(r-r_{0}\right)^{2}\right\} \\
E_{b e}(\theta)=\sum_{\text {node }}\left\{k_{\theta}\left(\theta-\theta_{0}\right)^{2}\right\} \\
E_{t o}(\phi)=\sum_{\text {node }}\left\{V_{1} \cos \phi+V_{2} \cos 2 \phi+V_{3} \cos 3 \phi+V_{6} \cos 6 \phi\right\}, \\
E_{v w}(\bar{r})=\sum_{\text {nonbonded }}\left\{A(\bar{r})^{-12}-C(\bar{r})^{-6}\right\}
\end{gathered}
$$

式(2) (5)中, $r_{0}$ 为键长的平衡位置, $\theta_{0}$ 为键角的平衡 位置, 而二面角有一个稳定状态 $\phi=180^{\circ}$ 和两个亚稳 定状态 $\phi= \pm 67.5^{\circ}$, 范德瓦尔势能平衡位置在 $\bar{r}=4.5$ $\mathrm{nm}$. 在模拟中各参数的选取如表 1 所示 ${ }^{[16]}$.

用于注射聚乙烯分子链的变截面纳米通道如图 2 所示, 它由一个体积为 $300 \AA \times 300 \AA \times 300 \AA$ 的正方
体(区域 $\mathrm{A}$ )通过一个棱台与一个横截面积为 $100 \AA \times$ $100 \AA$ 的长方体相连 (棱台与长方体为区域 $\mathrm{B}$ ), 区域 $\mathrm{A}$ 的左侧有一可移动壁面(图 2(b)). 系统初始化时, 10000 条聚乙烯分子的自由链被限制在区域 A 中, 每 条链由 30 个粒子构成, 即聚乙烯分子链的链长为 30 , 系统初始密度为 $0.2588 \mathrm{~g} \cdot \mathrm{cm}^{-3}$. 在进行注射过程的 模拟前, 首先对系统进行初始松他使系统达到平衡 状态; 之后, 给左侧可移动壁面施加一 $x$ 正方向的速 度, 同时给系统中的每一个粒子施加外力, 从而完成 注射过程. 这里采用施加外力场的方法代替压力梯 度, 是因为在外力场的作用下系统能更好地保持纵 向均衡 ${ }^{[18]}$. 通道壁面为虚拟热壁面, 整个模拟过程 通过速度修正法使系统的温度保持在 $450 \mathrm{~K}$, 采用

Verlet 数值积分法, 计算时间步长为 $1 \mathrm{fs}$.

为了研究微纳米通道结构对聚乙烯分子注射过 程的影响, 选取图 3 所示的 4 种纳米通道结构进行模 拟, 其相应的雉面倾角为 $\alpha=45^{\circ}, \alpha=71.6^{\circ}, \alpha=$ $78.7^{\circ}$ 和 $\alpha=90^{\circ}$.

聚合物链的回转半径是判断聚合链伸长和压缩 的重要参数, 常用于反映聚合物的流变特性. 本文 中, 聚乙烯链在流动方向 ( $x$ 方向)上的回转半径计算 如下:

$$
\left\langle R_{g x}^{2}\right\rangle=\frac{1}{M N} \sum_{p} \sum_{i}\left(r_{p i x}-r_{p g x}\right)^{2},
$$

式中, $r_{p i x}$ 表示第 $p$ 条链上的第 $i$ 个粒子的 $x$ 方向位置 $(i=1, \ldots, N$ 和 $p=1, \ldots, M), \quad r_{p g x}$ 表示第 $p$ 条链质心 $x$ 方向的位置, $r_{p g x}=\sum r_{p i x} / N, M$ 为分子链数, $N$ 为粒子数. 类似地, 聚乙烯链在 $y$ 和 $z$ 方向上的回转半径 $\left\langle R_{g y}^{2}\right\rangle$ 和 $\left\langle R_{g z}^{2}\right\rangle$ 也可根据相同的计算方法得到.

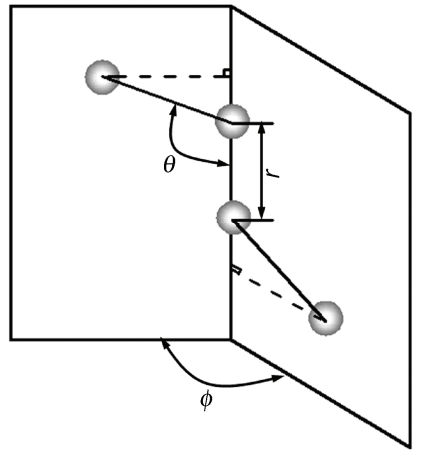

图 1 聚乙烯链简化模型示意图
表 1 势能参数表

\begin{tabular}{cc}
\hline 参数 & 数值 \\
\hline$r_{0}\left(\mathrm{~nm}^{-}\right)$ & 0.1533 \\
$k_{r}\left(\mathrm{~kJ} \mathrm{~mol}^{-1} \mathrm{~nm}^{-2}\right)$ & $1.373 \times 10^{5}$ \\
$\theta_{0}\left({ }^{\circ}\right)$ & 113.3 \\
$k \theta\left(\mathrm{kJ} \mathrm{mol}^{-1} \mathrm{~nm}^{-2}\right)$ & 374.7 \\
$V_{1}\left(\mathrm{~kJ} \mathrm{~mol}^{-1}\right)$ & 3.935 \\
$V_{2}\left(\mathrm{~kJ} \mathrm{~mol}^{-1}\right)$ & 2.177 \\
$V_{3}\left(\mathrm{~kJ} \mathrm{~mol}^{-1}\right)$ & 7.786 \\
$V_{6}\left(\mathrm{~kJ} \mathrm{~mol}^{-1}\right)$ & 0.0 \\
$A\left(\mathrm{~kJ} \mathrm{~mol}^{-1} \mathrm{~nm}^{-12}\right)$ & $2.972 \times 10^{19}$ \\
$C\left(\mathrm{~kJ} \mathrm{~mol}^{-1} \mathrm{~nm}^{-6}\right)$ & $6.907 \times 10^{9}$ \\
\hline
\end{tabular}




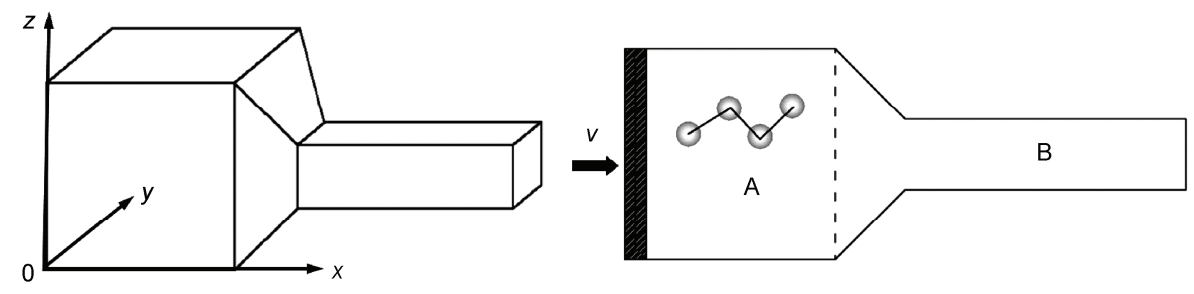

(a) (b)

图 2 注射用变截面纳米通道结构示意图

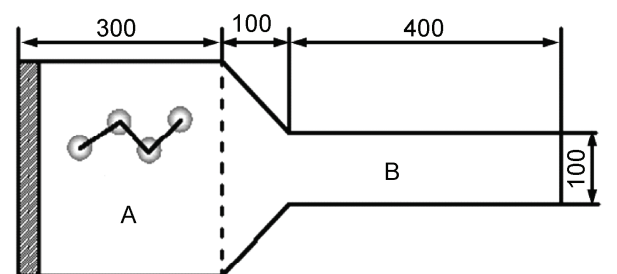

(a) $\alpha=45^{\circ}$

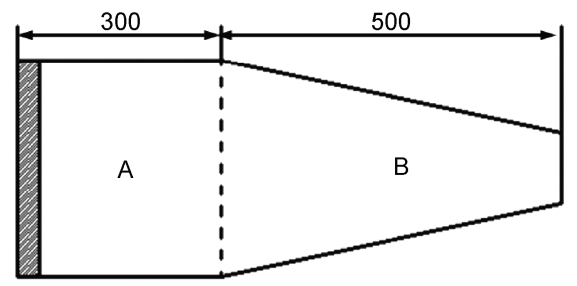

(c) $\alpha=78.7^{\circ}$

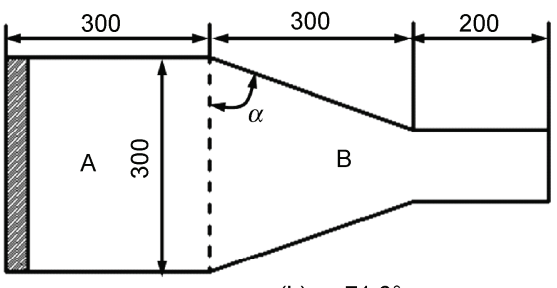

(b) $\alpha=71.6^{\circ}$

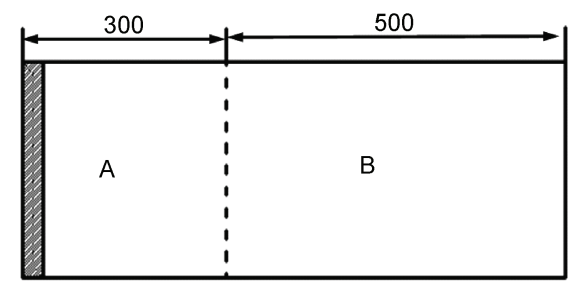

(d) $\alpha=90^{\circ}$

图 3 不同截面形状的纳米通道结构图(单位: ̊̊)

\section{2 结果及讨论}

\section{1 聚乙烯分子链的稳定特性}

在模拟注射过程前, 首先对每一种纳米通道区 域 A 中的聚乙烯分子链进行松驰直到系统达到稳定 状态，该松驰过程持续 20000 fs. 图 4 示出了聚乙烯 分子链在松弛 $20000 \mathrm{fs}$ 后其粒子数随键长、键角和二 面角的分布情况. 从图中可以看到在键长和键角的 平衡位置 $r_{0}=1.533 \AA$ 和 $\theta_{0}=113.3^{\circ}$ 处, 粒子数都出 现了峰值, 且粒子数在二面角 $\phi=67.5^{\circ}$ 和 $\phi=179^{\circ}$ 附 近也出现了两个峰值, 这说明大部分的分子链都已 处于平衡状态. 但值得注意的是, 在平衡位置 $r_{0}$ 和 $\theta_{0}$ 的附近仍有部分粒子出现, 这部分粒子具有很高 的能量, 这是因为在乙烯分子聚合成链的过程中, 分 子链结构之间相互限制, 这也使其二面角很难达到 绝对的稳态 $180^{\circ}$. 图 5 示出了松弛后区域 $\mathrm{A}$ 中聚乙
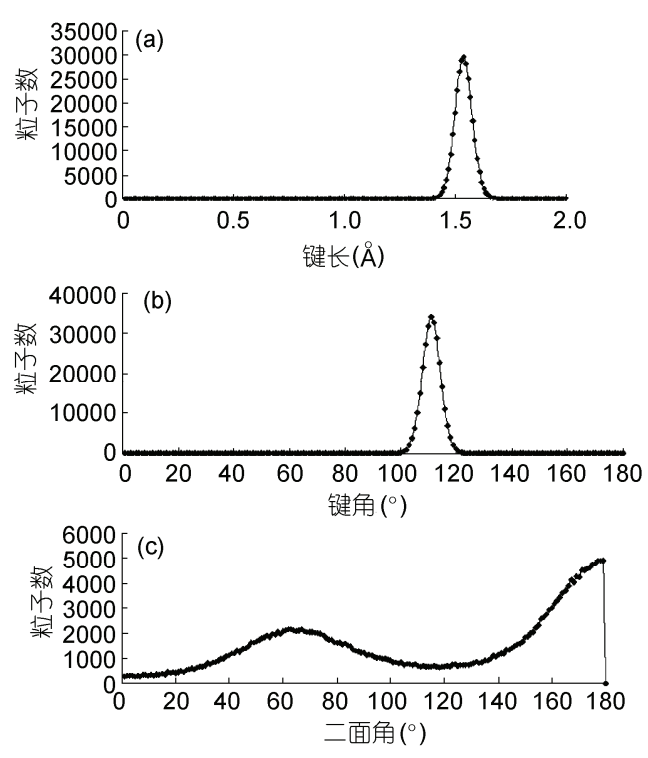

图 4 松驰 $20000 \mathrm{fs}$ 后聚乙烯分子各特征参数的分布 


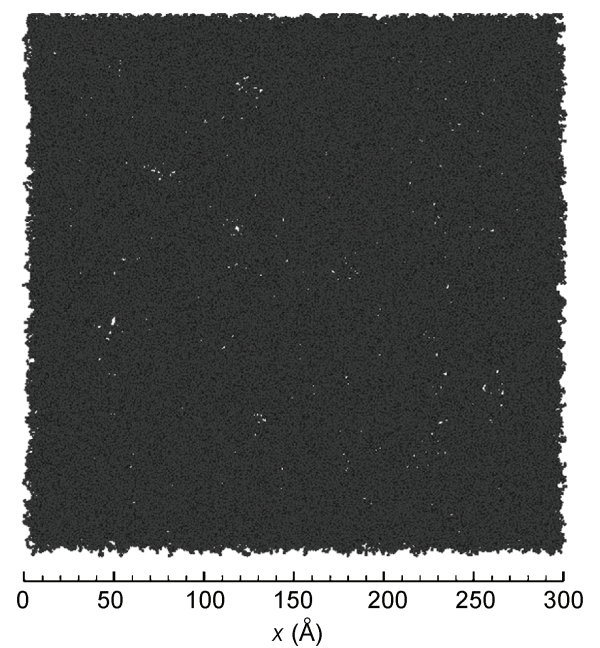

图 5 松驰后区域 A 中的聚乙烯分子链分布情况

烯分子链的分布情况, 图中黑色的微粒表示亚甲基 粒子. 从图中可以看到, 松驰后区域 A 中的粒子分布 出现了一些均匀的空隙.

\section{2 注射过程中聚乙烯流体的流变特性}

在松驰后, 给通道左侧可移动壁面施加一个 $x$ 正 方向的速度 $v=100 \mathrm{~m} \mathrm{~s}^{-1}$, 同时, 给系统中所有的亚甲 基粒子施加一个外力场 $f$ 推动聚乙烯流体向区域 $\mathrm{B}$ 流 动, 直至可移动壁面停在区域 $\mathrm{A}$ 与区域 $\mathrm{B}$ 的分界处, 模拟计算的总时间为 $320000 \mathrm{fs}$.

（i ）纳米通道结构对聚乙烯流体注射流动的影 响. 图 6 示出了以外加作用力 $f=20 \times 10^{-23} \mathrm{~J} \AA^{-1}$ 完 成注射过程时, 不同截面形状通道区域 $\mathrm{B}$ 中聚乙烯 分子链的分布情况. 从图中可以看出, 在外力场作用 下聚乙烯分子均流动到区域 $\mathrm{B}$ 中, 但是流动距离随 着通道截面形状的不同而不同. 在相同外力作用下,
随着通道雉面倾角减小, 聚乙烯分子流动距离增大, 区域 $\mathrm{B}$ 中聚乙烯流体能达到的最远位置分别为 $556.56 \AA, 576.23 \AA, 581.37 \AA$ 和 $587.78 \AA$. 这是由于 当聚乙烯分子链在外力作用下流动到区域 $\mathrm{B}$ 时, 随 着通道雉面倾角增大，虽然亚甲基粒子在流动方向 上受到的阻力逐渐减小, 但由于通道体积的增大, 导 致聚乙烯流体流动距离缩短, 聚乙烯分子链在单位 面积上的粒子密度也逐渐减小. 从图 6 中还观察到, 在锥面倾角 $\alpha=45^{\circ}$ 的纳米通道中, 注射过程结束时, 在松他过程后形成的均匀空隙全部消失了, 而在平 直纳米通道 $\left(\alpha=90^{\circ}\right)$ 中则存在大量不均匀空隙.

为了进一步了解流道内聚乙烯分子链的分布状 况和运动状态, 将流道由壁面到中心轴等分为 30 个 片层, 统计每一层分子链和亚甲基粒子的数目以及 平均速度, 可以得到通道内平均层密度和亚甲基粒 子的平均速度分布. 图 7 示出了聚乙烯分子链在坐标 $y$ 和 $z$ 方向上的分子链密度分布. 在四种通道中, 聚 乙烯分子链密度在 $y$ 和 $z$ 壁面上最小; 后快速升高并 出现了峰值, 且该峰值的大小以及其到壁面的距离 随着锥面倾角的减小而减小. 将分子链密度峰值所 在位置到壁面的距离定义为吸附层, 则从图中看到, 四种通道结构中, 锥面倾角为 $\alpha=45^{\circ}$ 的通道中吸附 层最薄, 吸附层内聚乙烯分子链的密度也最小, 此时 聚乙烯分子链在绝大部分通道空间内具有均匀的密 度分布. 此外, 图 8 还示出了聚乙烯分子链中亚甲基 粒子在坐标 $y$ 和 $z$ 方向上的密度分布. 与分子链密度 分布相似, 三种结构通道中, 亚甲基粒子密度分布也 均在接近 $y$ 和 $z$ 壁面附近出现了峰值, 且该峰值随着 锥面倾角的减小而逐渐靠近壁面. 但有所不同的是, 在雉面倾角为 $\alpha=45^{\circ}$ 的通道中, 亚甲基粒子密度分 布的最大值出现在 $y$ 和 $z$ 的壁面上, 并迅速下降至一

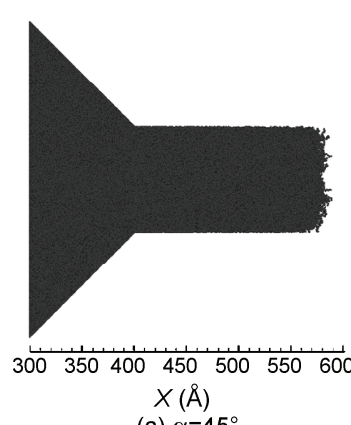

(a) $\alpha=45^{\circ}$

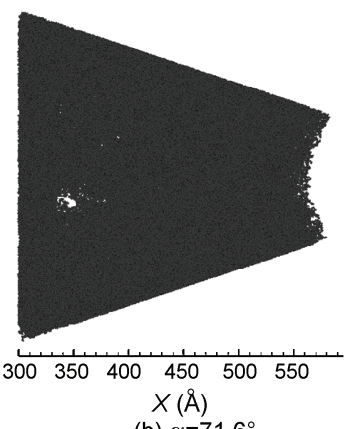

(b) $\alpha=71.6^{\circ}$

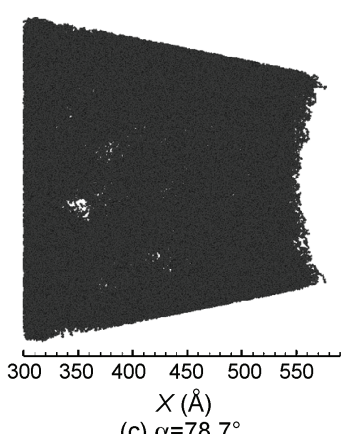

(c) $\alpha=78.7^{\circ}$

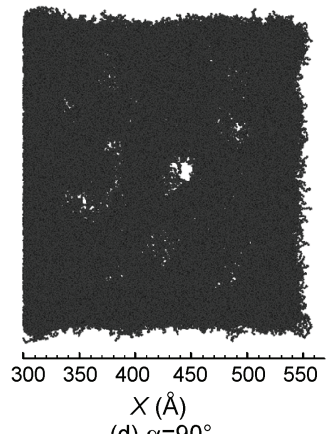

(d) $\alpha=90^{\circ}$

图 6 注射过程完成时区域 $\mathbf{B}$ 中的聚乙烯分子链分布情况 

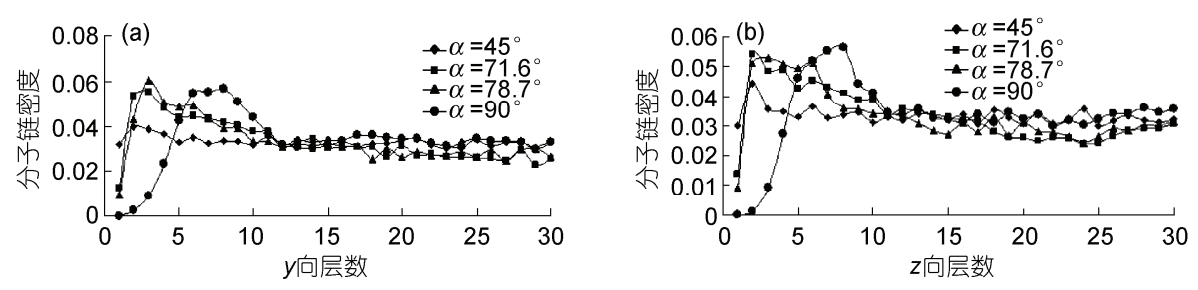

图 7 聚乙烯分子链在坐标 $y(a)$ 和 $z(\mathbf{b})$ 方向上的分子链密度分布
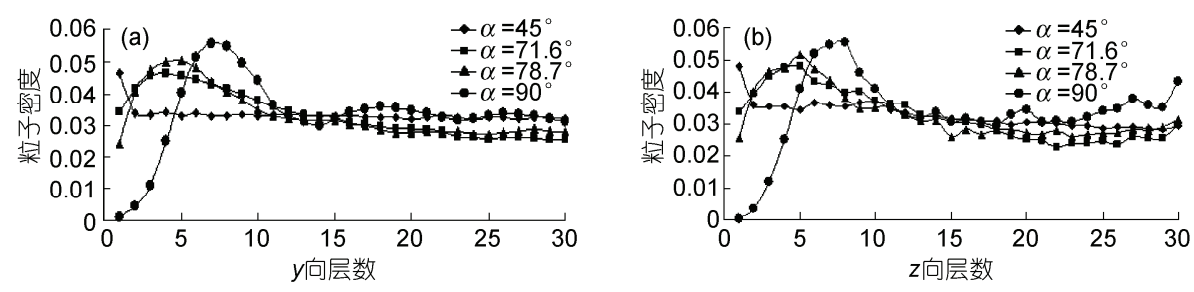

图 8 亚甲基粒子在坐标 $y(\mathbf{a})$ 和 $z(\mathbf{b})$ 方向上的粒子密度分布

稳定值, 然后在通道内绝大部分区域达到均匀分布. 从图 8 中还可以看到, 随着雉面倾角的增大, 吸附层 增厚, 吸附层内的粒子数增多, 这导致通道内粒子密 度的降低, 在雉面倾角 $\alpha=71.6^{\circ}$ 和 $\alpha=78.7^{\circ}$ 的通道 中, 通道内部的粒子密度均低于 $\alpha=45^{\circ}$ 的通道; 此 外, 在雉面倾角 $\alpha=90^{\circ}$ 的通道中, 吸附层最厚, 通道 内部的粒子密度变化波动较其他三种通道结构变化 大, 粒子分布不均匀. 因此, 无论是聚乙烯分子链的 密度分布还是亚甲基粒子的密度分布，模拟结果均 表明: 在雉面倾角为 $\alpha=45^{\circ}$ 的通道中聚乙烯分子充 分填充整个通道, 分布最为均匀, 没有非均匀的空隙 出现; 而在其他通道中, 由于各密度分布在通道内较 低、波动大，导致了空隙的出现.

图 9 示出了亚甲基粒子在坐标 $y$ 和 $z$ 方向上的粒 子平均速度分布. 在三种变截面通道中, 由于聚乙烯 分子链受到来自倾斜壁面的阻力, 粒子平均速度均 在靠近壁面处最小并逐渐增大. 沿着壁面向中心轴
方向, 四种通道中的粒子平均速度值越来越接近, 最 后趋于一个定值. 此外, 可以发现, 雉面倾角和变截 面通道长度对通道内粒子平均速度起着重要的影响, 较小的界面倾角和较长的变截面通道均将带来较大 的流动阻力, 从而导致流体流速减小. 在本模拟算例 中, 两者的综合作用导致粒子的平均速度在雉面倾 角 $\alpha=71.6^{\circ}$ 的通道中最小.

此外, 分子链的构型也会对聚乙烯流体流动产 生影响, 因而, 聚乙烯分子链的构型变化可从微观角 度反映聚乙烯流体的流动特性. 图 10(a)示出了松驰 过程结束后靠近壁面处某一条聚乙烯分子链的构型, 可以看到，聚乙烯分子链卷曲在一起，没有被拉伸。 图 10(b) (e)分别示出了注射过程结束后四种不同通 道中靠近壁面处同一条聚乙烯分子链的构型，可以 看到四种通道结构中聚乙烯分子都有不同程度的被 拉伸. 图 11(a)示出了松弛过程结束后通道中心处某 一条聚乙烯分子链的构型, 可以看到, 分子链构型在
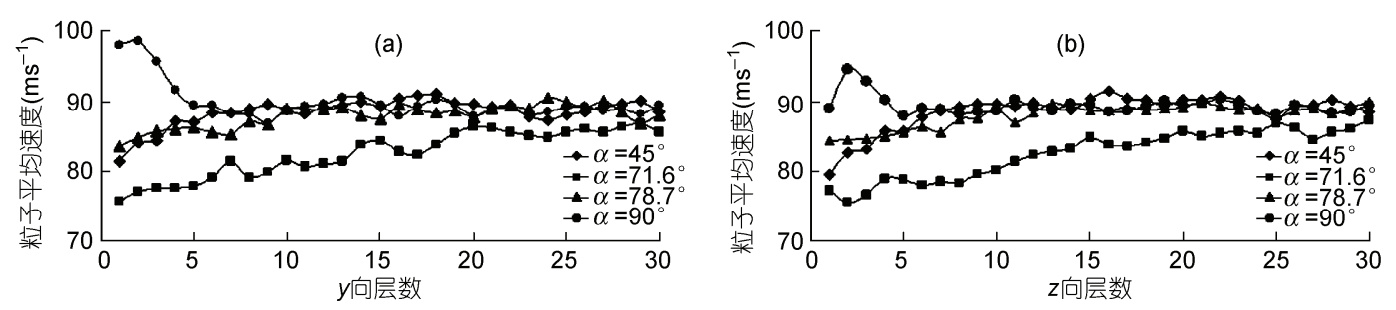

图 9 亚甲基粒子在坐标 $y(a)$ 和 $z(b)$ 方向上的平均速度分布 


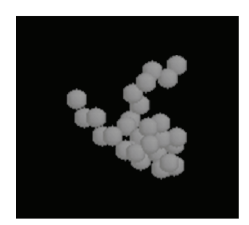

(a)

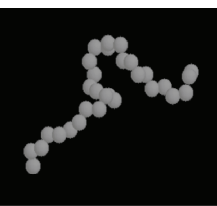

(b)

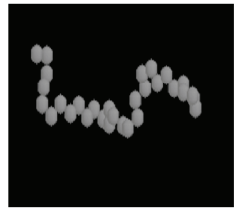

(d)

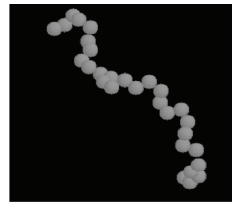

(c)

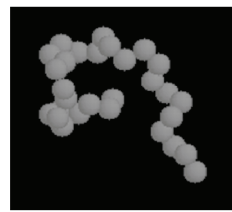

(e)
图 10 靠近壁面处随机聚乙烯分子链构型变化 (a) 注射前 (20000 fs); (b) (e) 注射后(320000 fs), 依次为 $\alpha=45^{\circ}$, $\alpha=71.6^{\circ}, \alpha=78.7^{\circ}$ 和 $\alpha=90^{\circ}$

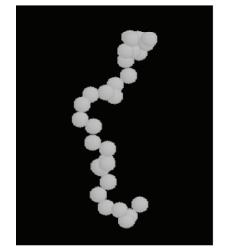

(a)

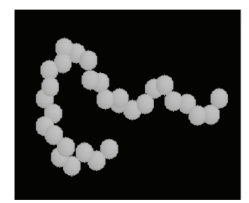

(b)

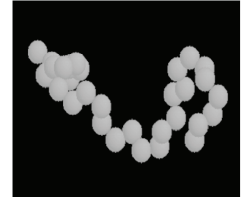

(d)

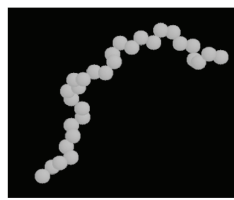

(c)

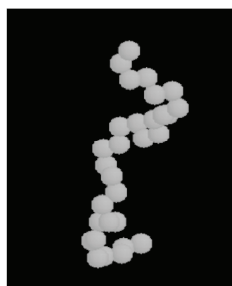

(e)
图 11 通道中心处随机聚乙烯分子链构型变化 (a) 注射前 (20000 fs); (b) (e) 注射后(320000 fs), 依次为 $\alpha=45^{\circ}$, $\alpha=71.6^{\circ}, \alpha=78.7^{\circ}$ 和 $\alpha=90^{\circ}$

流动方向没有被拉伸，而在 $z$ 方向上被拉伸，呈 I 字 型. 图 11(b) (e)分别示出了注射过程结束后四种不 同通道中通道中心处同一条聚乙烯分子链的构型. 在雉面倾角 $\alpha=45^{\circ}, \alpha=71.6^{\circ}, \alpha=78.7^{\circ}$ 三种通道结 构中，聚乙烯分子链在流动方向上都有不同程度的 被拉伸，但雉面倾角 $\alpha=90^{\circ}$ 通道结构中，聚乙烯分 子链的构型基本上没有变化, 说明雉面倾角 $\alpha=90^{\circ}$ 通道结构不利于聚乙烯流体在流动方向上的运动.

为了进一步分析聚乙烯分子链的拉伸情况，图 12 示出了不同结构通道中聚乙烯链在 $x, y$ 和 $z$ 方向上 的回转半径 $\left\langle R_{g x}^{2}\right\rangle,\left\langle R_{g y}^{2}\right\rangle$ 和 $\left\langle R_{g z}^{2}\right\rangle$ 随时间的变化. $\left\langle R_{g x}^{2}\right\rangle$ 的初始值为 $15.66 \AA^{2}$, 且由计算可知链长为 30
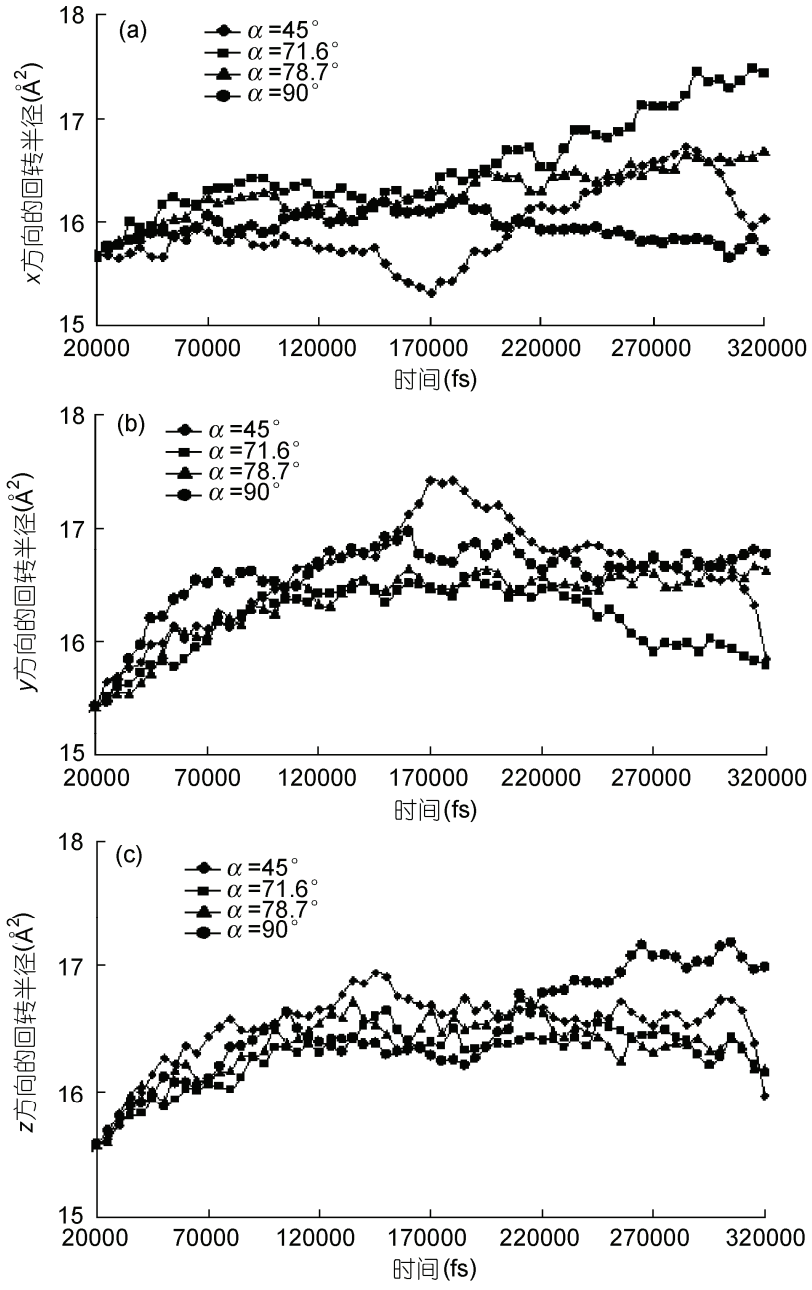

图 12 聚乙烯链在 $x, y$ 和 $z$ 方向上的回转半径 $\left\langle R_{g x}^{2}\right\rangle,\left\langle R_{g y}^{2}\right\rangle$ 和 $\left\langle R_{g z}^{2}\right\rangle$ 随时间的变化

的聚乙烯分子链完全拉伸时 $\left\langle R_{g x}^{2}\right\rangle$ 为 $176.06 \AA^{2}$, 由此 对比图 12 可知, 在四种通道内的注射过程中, 因为 几何的限制, 聚乙烯分子链均未完全扩展. 从图中还 可以看到,在雉面倾角 $\alpha=90^{\circ}$ 的通道中, $\left\langle R_{g x}^{2}\right\rangle$ 在注射 过程中变化不大, 说明分子链的拉伸和压缩现象均 不明显. 在锥面倾角 $\alpha=71.6^{\circ}$ 和 $\alpha=78.7^{\circ}$ 两种通道 中, $\left\langle R_{g x}^{2}\right\rangle$ 均随着注射时间逐渐增加而增大, 即在注 射过程中分子链在 $x$ 方向上被拉伸，且在 $\alpha=78.7^{\circ}$ 通 道中的拉伸更大. 而在倾角 $\alpha=45^{\circ}$ 的通道中, $\left\langle R_{g x}^{2}\right\rangle$ 的变化可分为四个阶段：注射初始时， $\left\langle R_{g x}^{2}\right\rangle$ 基本不 随时间变化; 接着, 在 $145000 \mathrm{fs}$ 后, 随着粒子开始进 人到区域 $\mathrm{B}$ 中的平直窄通道, $\left\langle R_{g x}^{2}\right\rangle$ 由于受到几何条 
件的限制而急剧减小; 随后, 在 $170000 \mathrm{fs}$ 时, 在流动 方向外力场的作用下, $\left\langle R_{g x}^{2}\right\rangle$ 又快速增大, 表明聚乙 烯分子链被拉伸; 最后, 在注射过程结束前, $\left\langle R_{g x}^{2}\right\rangle$ 再次减小, 此时分子链不再继续被拉伸, 而是逐渐被 压缩到接近回转半径的初始值. 此外, 在四种通道 中, 聚乙烯链在 $y$ 方向的回转半径 $\left\langle R_{g y}^{2}\right\rangle$ 在开始阶段 均随着时间的增加逐渐增大, 但随着粒子逐渐进人 到渐缩通道后, $\left\langle R_{g y}^{2}\right\rangle$ 出现不同的变化趋势. 在雉面 倾角 $\alpha=45^{\circ}$ 的通道中, $\left\langle R_{g y}^{2}\right\rangle$ 先快速增大并在 180000 fs 出现一个峰值, 之后 $\left\langle R_{g y}^{2}\right\rangle$ 随时间的增加而减小, 在 $305000 \mathrm{fs}$ 时大部分粒子进人到平直窄通道后, 由 于几何条件的限制, $\left\langle R_{g y}^{2}\right\rangle$ 的值急剧减小. 在雉面倾 角 $\alpha=71.6^{\circ}$ 的通道中, $\left\langle R_{g y}^{2}\right\rangle$ 随着时间变化先增加后 趋于平缓, 在粒子进人到渐缩通道后再逐渐减小. 而 在雉面倾角 $\alpha=78.7^{\circ}$ 和 $\alpha=90^{\circ}$ 的通道中, $\left\langle R_{g y}^{2}\right\rangle$ 随着 时间先增加, 然后趋于稳定. 对于聚乙烯链在 $z$ 方向 的回转半径 $\left\langle R_{g z}^{2}\right\rangle$, 除了其在雉面倾角 $\alpha=90^{\circ}$ 的通道 中随时间的增大而增大外, 在其他三种通道中, $\left\langle R_{g z}^{2}\right\rangle$ 均随时间的增大先增大而后趋于平衡. 由此, 我们可以得出在雉面倾角 $\alpha=45^{\circ}$ 的纳米通道中, 聚 乙烯分子链进人到区域 $\mathrm{B}$ 中平直窄通道后的一段时 间内出现了明显的沿流动方向的单轴拉伸现象, 这 更有利于分子链的有序输送.

(ii) 外加作用力对纳米通道内聚乙烯流体流动 的影响. 由上分析可知, 在雉面倾角 $\alpha=45^{\circ}$ 的纳米通 道中聚乙烯分子分布最为均匀, 且出现了单轴拉伸 现象, 有利于分子链的有序输送, 因此, 本节针对该 结构纳米通道研究外加作用力对聚乙烯流体流动的 影响, 模拟中外加作用力分别设定为 ${ }^{[19]} f_{1}=10 \times 10^{-23} \mathrm{~J}$ $\AA^{-1}, f_{2}=10 \times 10^{-23} \quad \mathrm{~J} \AA^{-1}$ 和 $f_{3}=20 \times 10^{-23} \mathbf{J} \AA^{-1}$.

图 13 示出了在不同外力作用下, 注射过程进行 了 $320000 \mathrm{fs}$ 时纳米通道中聚乙烯分子链的分布. 由图 可知, 聚乙烯流体在外力作用下流动到区域 $\mathrm{B}$ 中, 流 动的距离随着外加作用力的增大而增大. 三种作用 力下, 聚乙烯流体停止流动时的 $x$ 位置分别为 462.8 $\AA, 473.9 \AA$ 和 $587.8 \AA$, 流动距离增加的幅度随着作 用力的增大而显著提高, 且在注射过程结束时, 松弛 过程中形成的空隙均不再存在. 图 14 示出了亚甲基

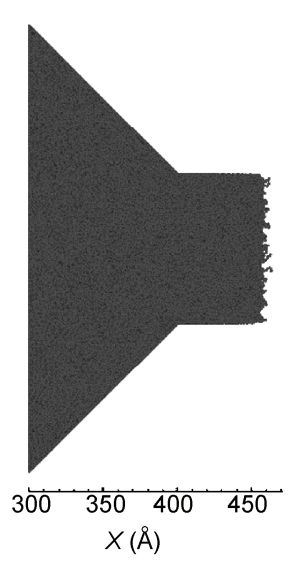

(a) $f_{1}$

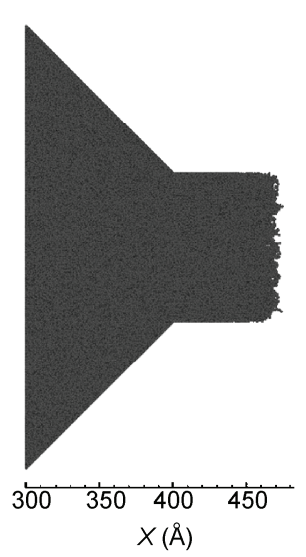

(b) $f_{2}$

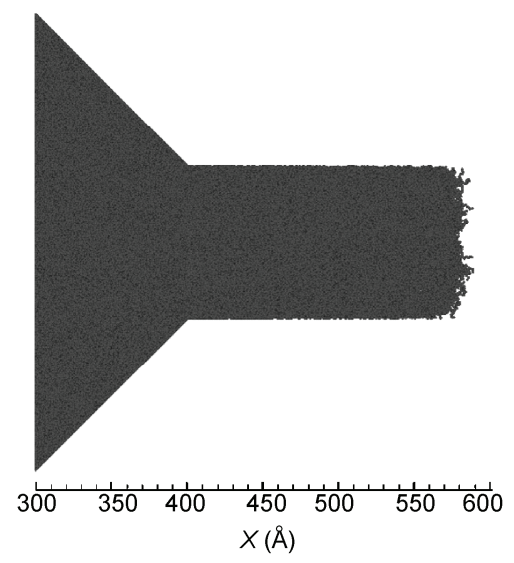

(c) $f_{3}$

图 13 注射过程完成时 $\alpha=45^{\circ}$ 通道中的聚乙烯分子链分布情况图
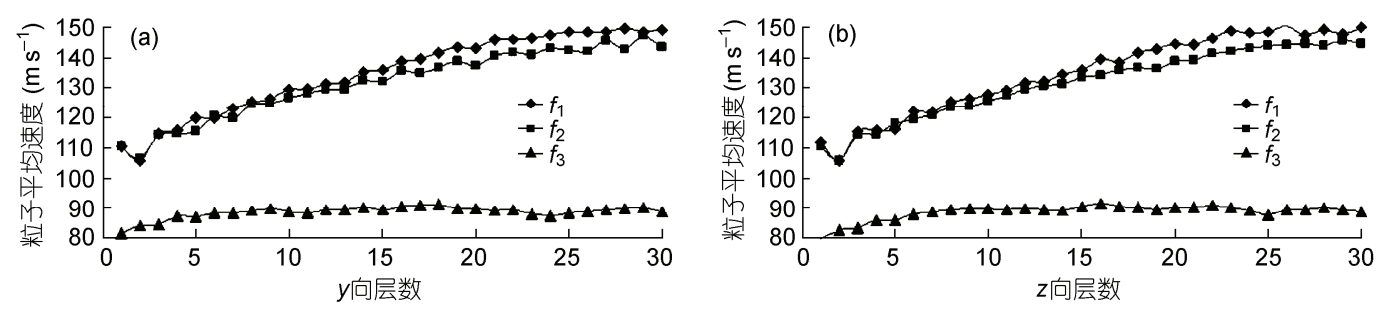

图 14 亚甲基粒子在坐标 $y$ 和 $z$ 方向上的平均速度分布 
粒子在坐标 $y$ 和 $z$ 方向上的粒子平均速度分布. 由图可 知, 在三种作用力情况下, 粒子平均速度均在壁面附 近处最小并逐渐增大, 而粒子平均速度随着作用力 的增大而减小, 在外力 $f_{3}$ 作用下, 粒子平均速度最小.

图 15 和图 16 分别示出了不同外力作用下, 注 射过程前后靠近壁面处某一条聚乙烯分子链和通道 中心处某一条聚乙烯分子链的构型变化. 在松弛过 程结束时,近壁面处聚乙烯分子链卷曲在一起, 没有 被拉伸(见图 15(a)), 而通道中心处聚乙烯分子链则

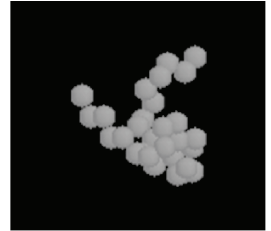

(a)

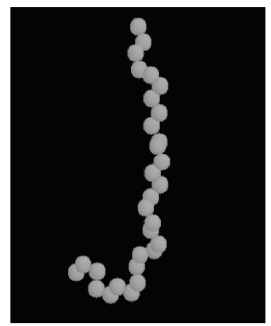

(c)

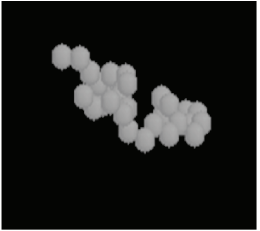

(b)

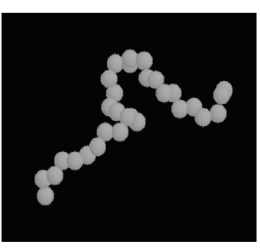

(d)
图 15 靠近壁面处随机聚乙烯分子链构型变化: (a) 注射前(20000 fs); (b) (d) 注射后(320000 fs), 依次为外力 $f_{1}, f_{2}$ 和 $f_{3}$

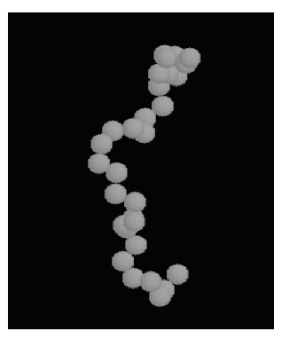

(a)

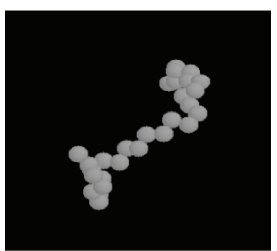

(c)

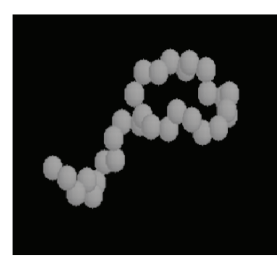

(b)

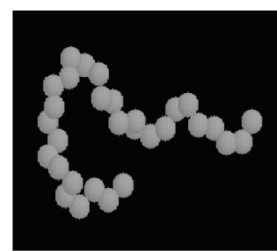

(d)
图 16 通道中心处随机聚乙烯分子链构型变化: (a) 注射前(20000 fs); (b) (d) 注射后(320000 fs), 依次为外力 $f_{1}, f_{2}$ 和 $f_{3}$
在 $z$ 方向上被拉伸，呈 I 字型(见图 16(a)). 注射过程 完成后, 可以看到聚乙烯分子都有不同程度的被拉 伸, 而在外力 $f_{3}$ 作用下, 分子链在流动方向上的拉 伸最明显(见图 15(b) (d)和图 16(b) (d)), 此时大部 分分子链已经与流动方向平行, 这有利于分子链有 序地进人到区域 B 中, 完成注射过程. 对比图 13 和 14 中发现, 在外力 $f_{3}$ 作用下, 粒子平均速度最小, 而流动距离却最远，这是由于在聚乙烯流体流动过 程中, 外力的作用除了推动流体流动之外, 还将造成 流体分子链构型的变化, 进而影响流动阻力特性及 流动距离. 分子链沿垂直于流动方向上的拉伸既阻 碍流体的流动，又降低了流体分子链在流动方向上 的占位; 而沿流动方向的拉伸将降低流体流动阻力, 同时又极大地增加了流体分子链在流动方向上的占 位. 因此，虽然外力 $f_{3}$ 作用下粒子平均速度最小， 但其分子链在流动方向上的显著拉伸使得其移动距 离却最大.

图 17 示出了聚乙烯分子链分别在 $x, y$ 和 $z$ 方向上 回转半径 $\left\langle R_{g x}^{2}\right\rangle,\left\langle R_{g y}^{2}\right\rangle$ 和 $\left\langle R_{g z}^{2}\right\rangle$ 随时间的变化. 从图中
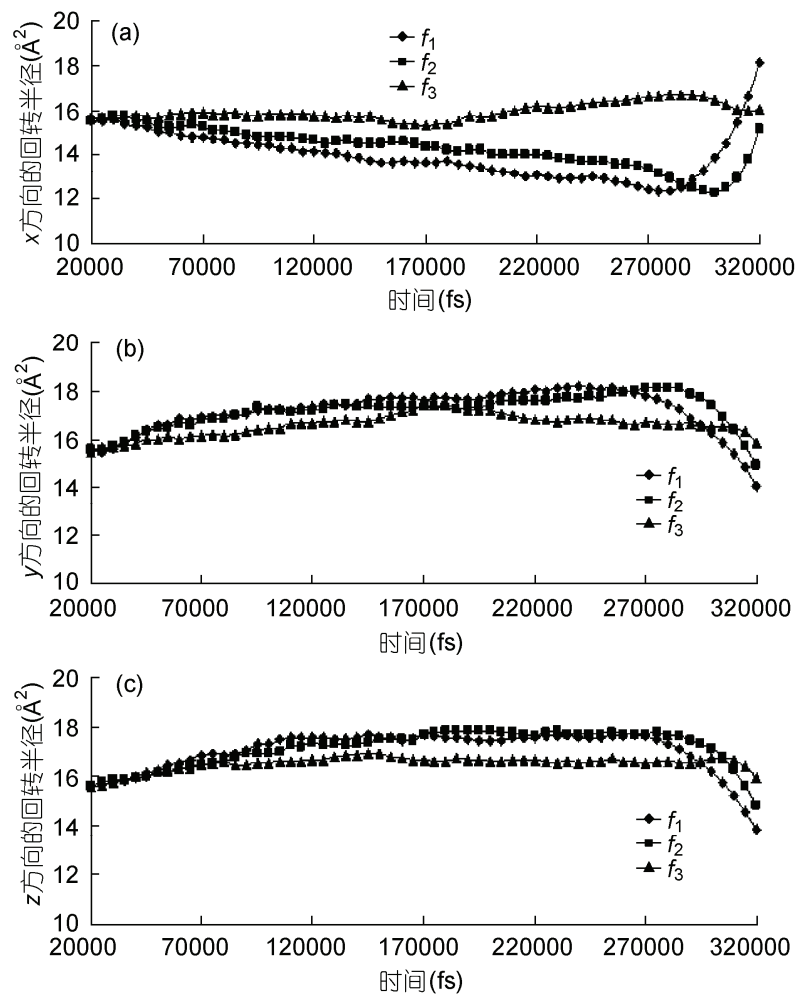

图 17 不同外加作用力下 $\alpha=45^{\circ}$ 通道中聚乙烯分子链的回 转半径 $\left\langle\boldsymbol{R}_{g x}^{2}\right\rangle,\left\langle\boldsymbol{R}_{g y}^{2}\right\rangle$ 和 $\left\langle\boldsymbol{R}_{g z}^{2}\right\rangle$ 随时间的变化 
可以看到, 在外力 $f_{1}$ 和 $f_{2}$ 作用下, 聚乙烯分子链在三 个方向上的回转半径均有相似的变化规律, 并有别 于外力 $f_{3}$ 下的情况. 在 $f_{1}$ 和 $f_{2}$ 作用下, 由于区域 B 中棱雉斜面对聚乙烯流体的阻力作用, $\left\langle R_{g \mathrm{r}}^{2}\right\rangle$ 被逐步 压缩; 当流体粒子逐渐进入到平直窄通道后, $\left\langle R_{g x}^{2}\right\rangle$ 转而升高, 聚乙烯分子链逐步恢复到接近松驰后的 初始值. 但 $\left\langle R_{g x}^{2}\right\rangle$ 在 $f_{3}$ 作用下变化并不明显, $170000 \mathrm{fs}$ 后, 聚乙烯分子链被逐渐拉伸最后也恢复到接近初 始值. 此外还可以看到在 $f_{1}$ 和 $f_{2}$ 外力作用下, 回转半 径 $\left\langle R_{g y}^{2}\right\rangle,\left\langle R_{g z}^{2}\right\rangle$ 均先随着时间的增加而略有增大; 当 流体粒子进人区域 B 中的平直窄通道后, $\left\langle R_{g y}^{2}\right\rangle 、\left\langle R_{g z}^{2}\right\rangle$ 由于几何条件的限制而减小. 而在 $f_{3}$ 外力作用时, $\left\langle R_{g y}^{2}\right\rangle,\left\langle R_{g z}^{2}\right\rangle$ 变化均不明显. 由此可以得出, 在 $f_{3}$ 外 力作用下，聚乙烯分子链仅在流动方向被逐渐拉伸， 使得其更容易流动到区域 $\mathrm{B}$ 中, 从而达到注射要求.

\section{3 结论}

本文采用分子动力学方法对聚乙烯分子链在变 截面纳米通道中注射流动的流变特性进行了研究, 探讨了通道结构和外加作用力对纳米通道内聚乙烯 流体注射流动的影响, 得到以下主要结论: (1)靠近 壁面存在粒子吸附层, 且吸附层的厚度随通道雉面 倾角的增大而增大; (2)在锥面倾角为 $\alpha=45^{\circ}$ 的变 截面纳米通道中, 聚乙烯分子链分布最为均匀, 且 在注射过程中出现了沿流动方向的单轴拉伸现象, 降低了流动阻力, 增加了分子链在流动方向上的占 位; (3)注射过程中, 聚乙烯分子链在变截面纳米通 道中的运动距离随着雉面倾角减小及外加作用力增 大而增大. 在本文模拟研究范围内, 在雉面倾角为 $\alpha=45^{\circ}$ 的纳米通道中施加外力 $f=20 \times 10^{-23} \mathrm{~J} \AA^{-1}$ 更 有利于分子链有序地进人到变截面通道中完成注射 过程.

\section{参考文献}

1 Gorbounov V, Kuban P, Dasgupta P K, et al. A nanoinjector for microanalysis. Anal Chem, 2003, 75: 3919-3923

2 Zeng H L, Seino N, Nakagma T, et al. Accurate nano-injection system for capillary gas chromatography. J chromatogr A, 2009, 1216: $3337-3342$

3 Kim S W. Debinding behaviors of injection molded ceramic bodies with nano-sized pore channels during extraction using supercritical carbon dioxide. J Supercrit Fluids, 2010, 51: 339-344

4 Berkland C, Pack D W, Kim K K. Controlling surface nano-structure using flow-limited field-injection electrostatic spraying (FFESS) of poly(D, L-lactide-co-glycolide). Biomaterials, 2004, 25: 5649-5658

5 Lgnjatovic N L, Liu C Z, Czernuszka J T, et al. Micro- and nano-injectable composite biomaterials containing calcium phosphate coated with poly(D, L-lactide-co-glycolide). Acta Biomater, 2007, 3: 927-935

6 Wimberger -Friedl R. The assessment of orientation, stress and density distributions in injection-molded amorphous polymers by optical thechniques. Prog Polym Sci, 1995, 20: 369-401

7 Khor C Y, Ariff Z M, Che Ani F, et al. Three-dimensional numerical and experimental investigations on polymer rheology. Int Communications Heat Mass Transfer, 2010, 37: 131-139

8 Rudisill J W, Cummings P T. Non-equilibrium Molecular dynamics approach to the rheology of model polymer fluids. Fluid Phase Equilib, 1993, 88: 99-113

9 Daivis P J, Matin M L, Todd B D. Nonlinear shear and elongational rheology of model polymer melts at low strain rates. J Non-Newtonian Fluid Mechanics, 2007, 147: 35-44

10 Hess S, Rheological properties via nonequilibrium molecular dynamics: From simple towards polymeric liquids. J Non-Newtonian Fluid Mech, 1987, 23: 305-319

11 Jabbarzadeh A, Atkinson J D, Tanner R J. Nanorheology of molecularly thin films of n-hexadecane in Couette shear flow by molecular dynamics simulation. J Non-Newtonian Fluid Mech, 1998, 77: 53-78

12 Yung K L, He L, Xu Y, et al. Study of surface conditions and shear flow of LCP melts in nanochannels through molecular dynamics simulation. Polym, 2006, 47: 4454-4460

13 Herrchen M, Ottinger H C. A detailed comparison of various Fene dumbbell models. J Non-Newtonian Fluid Mech, 1997, 68: 17-42

14 Kramers H A. The viscosity of macromolecules in a streaming fluid. Physica 1944, 11: 1-19

15 Rouse P E. A theory of the linear viscoelastic properties of dilute solutions of coiling polymers. J Chem Phys, 1953, 21: 1272-1280

16 Yashiro K, Ito T, Tomita Y. Molecular dynamics simulation of deformation behavior in amorphous polymer: nucleation of chain entanglements and network structure under uniaxial tension. Mech Sci, 2003, 45: 1863-1876

17 Leach A R. Molecular Modelling: Principles and Applications. 2nd ed. Singapore: Addison Wesley Long-man, 2001

18 Travis K P, Todd B D, Evans D J. Poiseuille flow of molecular fluids. Phys A, 1997, 240: 315-327

19 Rapaport D C. The Art of Molecular Dynamics Simulation. 2nd ed. London: Cambridge University Press, 1995 